$20 \mid 1999$

Varia

\title{
Les résonances actuelles de la « raison des effets »
}

\section{Philippe Ducat}

\section{OpenEdition}

\section{Journals}

Édition électronique

URL : http://journals.openedition.org/ccibp/554

DOI : $10.4000 /$ ccibp.554

ISSN : 2493-7460

\section{Éditeur}

Centre international Blaise Pascal

\section{Édition imprimée}

Date de publication : 19 octobre 1999

Pagination : 55-63

ISSN : 0249-6674

Référence électronique

Philippe Ducat, « Les résonances actuelles de la « raison des effets » », Courrier du Centre international Blaise Pascal [En ligne], 20 | 1999, mis en ligne le 06 janvier 2016, consulté le 30 avril 2019. URL: http://journals.openedition.org/ccibp/554; DOI : 10.4000/ccibp.554

Ce document a été généré automatiquement le 30 avril 2019.

Centre international Blaise Pascal 


\title{
Les résonances actuelles de la « raison des effets 》
}

\author{
Philippe Ducat
}

«Jusqu'où peut-on interpréter?", demandait ici-même Jean Mesnard. C'est la question que je serais tenté de poser à un certain nombre de nos contemporains : ceux d'entre eux qui entreprennent de rendre compte de la pensée politique de Pascal, tout d'abord, et s'appuient généralement dans une large mesure, pour ce faire, sur les fragments de la liasse $\mathrm{V}$ (et les textes connexes, comme les fragments 60, 525 et 828 de l'édition Lafuma) ou, aujourd'hui, le Discours XV de l'édition Martineau. Mais cette question peut tout aussi légitimement être adressée, au-delà, aux "philosophes et savants » qui voient dans l'application pascalienne des schèmes de la « raison des effets » au champ socio-politique, non un objet d'étude, mais une source d'inspiration: tous ceux, en somme, qui considèrent aujourd'hui avec Pierre Bourdieu que l'on peut « lire comme un programme de travail scientifique et politique » tel ou tel « texte fameux sur « la raison des effets ${ }^{1}$ ", que ce soit pour y trouver, comme le sociologue, un renfort possible dans la définition de concepts opératoires des sciences sociales («champ», «disposition», "pouvoir symbolique », par exemple), ou pour y dépister, à l'instar de Jacques Derrida, l'idée ou le pressentiment d'une justice irréductible au droit ou à la contrainte légale, et pourtant non dépourvue de force. Resterait alors aux exégètes à déterminer ce qui, dans le texte pascalien lui-même, appelle ou rend possible une telle diversité de "stratégies " d'appropriation, d'exploitation ou de reformulation.

\section{I - Une pensée politique en débat}

2 Y a-t-il une pensée politique de Pascal ? Ce présupposé de l'interprétation que proposait Gérard Ferreyrolles en $1984^{2}$ n'est guère susceptible d'être remis en question. Mais cette «politique de Pascal» suscite, dès lors qu'on s'efforce de l'élucider et de l'évaluer, un conflit d'interprétations. Non sans quelque artifice, on pourrait reconstituer les discussions actuelles en les regroupant sous trois têtes de chapitres: la modernité de 
Pascal, appréciée à l'aune du débat entre normativisme et décisionnisme juridiques, l'autonomie qu'il reconnaît à la sphère politique, et enfin le problème résiduel de la cohérence doctrinale et des effets politiques possibles de sa pensée politique.

\section{1/ Une politique moderne?}

3 Qu'on l'approuve pour son réalisme ${ }^{3}$ ou que l'on stigmatise son immoralisme ${ }^{4}$ (mais Gérard Ferreyrolles estime pour sa part que la politique de Pascal déplaît fatalement à tous ${ }^{5}$ ), le texte pascalien fait l'objet d'investissements exégétiques qui sont à la mesure de l'actualité des questions qu'il traite et des réponses qu'il y apporte - ce qui pourrait conférer à leur interprétation un caractère lui-même politique, donc polémique. Pascal, miroir de la modernité ? "La politique de Pascal nous renvoie nos propres concepts", affirme Gérard Ferreyrolles ${ }^{6}$. A tout le moins, on pourrait considérer que cette « politique » contribue à situer et éclairer certains de nos débats : celui, par exemple, qui met aux prises normativisme et décisionnisme.

Peut-on imaginer qu'en dépit du rôle qu'il accorde à la coutume (qui fait «toute l'équité ", fr. 60) et de son pessimisme anthropologique, Pascal croie possible d'ordonner la décision et l'action politique à des normes prédéfinies? Tout dépend du sens accordé au fr. 60 de l'édition Lafuma: en énonçant «il y a sans doute des lois naturelles, mais cette belle raison corrompue a tout corrompu ", Pascal disqualifie-t-il d'avance tout recours au substrat normatif que pourrait fournir la «loi naturelle», voire un hypothétique «droit naturel » qui en dépendrait, ou insiste-t-il au contraire sur la force d'obligation intacte, et donc la pertinence morale et politique, en dépit du péché et de toute l'histoire humaine, de ces « lois naturelles » ineffaçablement gravées dans le cœur de l'homme par son bienveillant créateur ? Pierre Magnard ${ }^{7}$ avait vigoureusement écarté cette dernière hypothèse. "De droit naturel point [chez Pascal] ", écrivait-il il y a plus de vingt ans, " ni au sens dogmatique de Suarez, ni au sens critique de Grotius, ni au sens moyen de Domat et de Nicole. [...] Cette remise en question du fondement du droit s'effectue en trois étapes: critique de la notion de loi naturelle, antinomie justice-force, renversement du pour au contre. Ce cheminement peut passer par Bodin et Montaigne, Machiavel et Grotius; il n'en est pas moins absolument original par le type de raisonnement auquel il introduit et que Pascal désigne de ces simples mots: "La raison des effets" ".

Or sur ce point crucial les travaux plus récents de Gérard Ferreyrolles prétendent modifier fondamentalement le regard du lecteur. La force et la coutume, à l'en croire, ne sauraient selon Pascal tout justifier; qu'il faille obéir aux lois parce qu'elles sont lois - simple « conseil de prudence politique ${ }^{8}$ » du fr. 525 - ne signifierait pas qu'on soit tenu d'obéir à une loi injuste. Que Pascal affirme qu'« il y a sans doute des lois naturelles » équivaudrait donc de sa part à une prise de position normativiste (voire anti-absolutiste : « Il existe pour Pascal des normes de justice que l'autorité établie est tenue de respecter ${ }^{9}$ »), plus précisément jusnaturaliste : «La force ne [...] légifère validement qu'à l'intérieur des limites posées par le droit naturel ou divin [...], l'établissement [...] ne fait la justice d'une loi qu'à la condition que le droit naturel ou divin ait auparavant justifié l'établissement [...]. Se soustraire à l'observation de la loi naturelle, c'est, pour un État, s'exclure de l'existence ${ }^{10}$ ». S'il y a des lois naturelles, la coutume ne fait donc pas, à proprement parler, toute l'équité. Ou plutôt, il faut voir dans son équité, sinon une émanation, du moins une imitation de l'essentielle justice : « La coutume apparaît en fait 
le réceptacle de la loi naturelle, comme elle non écrite et antérieure aux lois édictées par les hommes [...] Si la paix règne dans les communautés humaines, c'est que la coutume les modèle en quelque façon à l'image de l'origine antélapsaire ${ }^{11}$ ».

6 Originale et radicale, cette lecture de Pascal ne manque pas de prêter le flanc à la critique. D'un point de vue simplement méthodologique, il peut sembler imprudent d'accorder le même statut et la même importance aux Pensées et aux Provinciales (notamment les Lettres quatorze et quinze, très sollicitées par Gérard Ferreyrolles). Là, Pascal expose ses positions les plus personnelles; ici il tâche de se montrer aussi proche que possible des doctrines communément reçues dans l'Église. Surtout, les premières lignes de la Lettre quatorze donnent l'impression que Pascal traite dans ce texte de la loi révélée (à Noé et Moïse, pour commencer) davantage que de la loi naturelle, ou a fortiori d'un hypothétique «droit naturel ». Au demeurant, on a parfois l'impression que le texte des Pensées est lui aussi de temps à autre malmené par l'interprétation de Gérard Ferreyrolles. Citant, par exemple, le fr. 148 qui réfute le suicide « contraire à Dieu, à la raison et à la nature tout ensemble », cet exégète veut y voir une preuve que selon Pascal « la loi naturelle suppose la concupiscence et l'ordonne", ou pour mieux dire que "la loi naturelle réconcilie le désir avec la raison ${ }^{12} »$. Or Pascal dans ce fragment s'efforce précisément de montrer que l'homme peut extirper de son cœur le désir de persévérer dans son être ${ }^{13}:$ «Depuis qu'il a perdu le vrai bien tout également peut lui paraître tel jusqu'à sa destruction propre »; de sorte que si Pascal mentionne ici «la nature", c'est pour montrer que sa loi ne nous oblige plus effectivement à coup sûr, ou que si elle a été gravée dans nos cœurs elle n'y a guère laissé de vestige déchiffrable. Par contrecoup, on peut alors être tenté de revenir sur l'interprétation du fr. 60 qu'a proposée Gérard Ferreyrolles; et lui objecter avec Christian Lazzeri que l'obligation où chacun se trouve de "[suivre] les lois de son pays " atteste que la loi naturelle, même si elle existe, n'est pas actuellement connue de l'homme, et que tout au plus elle subsiste dans notre nature déchue « comme une trace illisible de notre condition antélapsaire ${ }^{14} »$. Impossible dès lors de fonder sur l'invocation d'une "loi naturelle» dont l'écho est à ce point assourdi un normativisme politique : comme le souligne Christian Lazzeri, «il ne sert à rien, pour régler normativement les comportements, que les lois naturelles existent si elles ne sont pas connues naturellement ${ }^{15} \gg$.

7 Qu'opposer au normativisme juridique et politique? De prime abord, on pourrait être tenté de rapprocher Pascal des modernes positivistes: le penseur de la coutume et de l'établissement ne leur accorderait-il pas que le droit se définit par le fait de sa promulgation et de sa reconnaissance (reposât-elle sur la croyance en sa légitimité), plutôt que par son adéquation aux normes d'une idéale justice ? Christian Lazzeri suggère ainsi que pour Pascal « la justice (ou le droit) positive que ne légitime aucune loi naturelle connaissable se réduit [...] au fait de la loi elle-même ${ }^{16}$ ». À y regarder de plus près, cependant, il apparaît que Pascal ne dissocie pas la prise en compte du fait de la loi d'une réflexion plus fondamentale sur les conditions et les modalités de son institution, c'est-àdire sur le jeu des forces qui donnent à la loi force de loi. Les fragments 85 (« l'épée donne un véritable droit») et 828 ( «ils battront jusqu'à ce que la plus forte partie opprime la plus faible ») assignent explicitement pour origine à la loi et à l'ordre la décision appuyée sur la force. Pascal pourrait-il être reconnu par Carl Schmitt comme l'un des précurseurs de son décisionnisme juridique? Ce rapprochement peut d'abord être justifié par une mise en perspective historique de la réflexion politique de Pascal. Comme, dans un autre champ, Corneille ou Racine, soulignait pour sa part Carl Schmitt dans son essai de 1942 
sur « la formulation de l'esprit française par les légistes », Pascal récolte « les fruits mûris pendant les années de guerre civile»: sa pensée politique prend forme au sein d'un royaume qui « opta pour le catholicisme, mais par une décision politique de l'État, décision faisant partie intégrante de l'ordre public ». Cette pensée repose ainsi « sur le socle d'une décision politique rendue possible par la souveraineté de l'État ${ }^{17}{ }^{\prime}$.

En outre, les exégèses contemporaines de Pascal soulignent volontiers certains traits proprement décisionnistes de sa pensée. On peut remarquer, pour commencer, que si le décisionnisme contemporain se définit par sa définition de la souveraineté comme pouvoir de décider de la situation exceptionnelle ${ }^{18}$, Pascal soulignait déjà quant à lui que le souverain est celui qui a seul l'obligation et la possibilité, pour assurer le salut de l'État, de suspendre la légalité constituée : notant tantôt qu'il «n'est pas étrange qu'un État subsiste lorsqu'on fait quelquefois céder ses lois à la nécessité", tantôt, et plus radicalement, que « les États périraient si on ne faisait ployer souvent les lois à la nécessité 19 ». Christian Lazzeri exploite ces passages comme autant d'indices de l'illégitimité d'une lecture normativiste de Pascal («les lois naturelles ne peuvent plus être invoquées comme limite à l'exercice du pouvoir des gouvernants $\left.{ }^{20} »\right)$, mais plus profondément il montre qu'il faut voir dans la décision de l'exception à la règle commandant de tenir secrètes les sources réelles d'un "droit " lui-même issu d'une décision politique: en décidant de la situation exceptionnelle, le souverain révèle en effet que «la force dispose des lois selon les décisions des gouvernants, qui peuvent les suspendre par le moyen même avec lequel ils les ont instituées ${ }^{21} »$. Cette exhibition, dans et par la décision de l'exception, de la nature et peut-être même de l'origine du droit, pose du reste le redoutable problème de la légitimation de cette décision éminente: impossible, pour le coup, de s'appuyer sur la coutume, ou sur la croyance des sujets en la justice des lois! Mais Christian Lazzeri vent croire qu'ici la légitimation, qui ne peut être fournie par aucun substrat normatif préconstitué, surgit de l'urgence même de la décision:» Le caractère exceptionnel de la situation [...] peut être publiquement [constaté] par les sujets, qui peuvent ainsi accepter les mesures requises ${ }^{22} »$. Peut-être ce commentateur prête-t-il ici aux sujets davantage d'habileté que Pascal ne leur en reconnaîtrait, et minore-t-il le rôle nécessaire de la contrainte dans l'exercice du pouvoir absolu, surtout dans les rares occasions où celui-ci se manifeste dans la plénitude de son essence, c'est-à-dire de sa force.

Bien entendu, on ne saurait pour autant imaginer que Pascal glorifie la force pure sans prendre en compte quelque exigence normative que ce soit. Son propos n'est certes pas de réduire le droit à l'arbitraire de la violence : après tout, relevait Jean Mesnard lors d'une discussion à Clermont-Ferrand en 1990, «la force n'est pas la violence ${ }^{23}$ ». Et Pierre Force peut même ajouter: «Force et justice ne sont pas antonymes, mais bien synonymes ${ }^{24}$ ». De fait, comme y insiste ce commentateur, c'est bien dans la mesure où « la force est très reconnaissable et sans dispute » (fr. 103) qu'elle contribue à façonner une justice terrestre reconnaissable. C'est d'être " arbitraires et non sujettes à examen ou à discussion " qui peut rendre les prescriptions de la force "naturelles et justes » aux yeux de ceux auxquels elles s'appliquent. Plus forte que toute norme préétablie, libérée, dirait Carl Schmitt, de toute obligation normative, la force est institutrice d'un ordre, et de critères du juste et de l'injuste : au même titre que la coutume, la force a ainsi selon Pierre Force «l'immense mérite d'établir des normes ${ }^{25}$ ». Qu'on n'aille pas croire que cette interprétation permette, en insistant sur le pouvoir nomothétique de la force, d'opposer Pascal au décisionnisme contemporain : bien au contraire, lorsque Carl Schmitt 
évoque la situation exceptionnelle où nulle norme reçue ne peut valoir («il n'existe pas de norme qu'on puisse appliquer à un chaos»), c'est pour souligner lui aussi que la décision politique, dans son "absolue pureté ", impose un ordre, rend possible «une organisation normale des conditions de vie ", et donc suscite la définition et l'application d'une « réglementation normative ». Est souverain celui qui non seulement décide de la situation exceptionnelle, mais encore « décide si [la] situation normale existe réellement ${ }^{26}$ ». L'originalité de Pascal serait peut-être, à cet égard, d'avoir thématisé au titre de la « coutume » et de « l'imagination » les mécanismes d'institutionnalisation progressive (et non seulement d'institution primitive) de cette situation « normale ».

\section{2/ L'autonomie du politique}

10 Même si ses parties prenantes n'en ont pas conscience ou ne jugent pas souhaitable de formaliser dans ces termes les présupposés et les enjeux polémiques de leurs analyses, il peut donc se révéler éclairant de tâcher d'inscrire le débat sur la pensée politique de Pascal au cœur de la discussion principielle, invitée par Carl Schmitt dans les années 1920, entre normativisme et décisionnisme politique. Schématiquement, on pourrait également déduire de la position ainsi adoptée quant à l'orientation fondamentale de la "politique " pascalienne le degré d'autonomie reconnu par Pascal, selon ses commentateurs, à la sphère politique, au regard notamment de l'ordre de la charité. La question se peut aisément formuler en termes techniques : en distinguant, au fr. 90, entre "le peuple», les "demi-habiles", les «habiles", les "dévots», et les "chrétiens parfaits», Pascal propose-t-il une gradation linéaire, qui finirait par dégager le point de vue du « chrétien parfait » sur la politique comme le plus pertinent et le seul vrai, ou esquisse-t-il en les juxtaposant deux séries de trois éléments, comportant chacune un changement d'ordre, et conduisant respectivement, la première, du peuple (charnel) aux habiles (qui atteignent à l'excellence dans l'ordre de l'esprit), dès lors dotés d'un point de vue sur la politique parfait dans son ordre, et la seconde des habiles aux deux catégories de croyants, dont la position ne pourrait apparaître comme supérieure qu'envisagée depuis un autre plan, non proprement politique, à savoir celui de la charité ? Pierre Magnard semblait opter pour la première hypothèse lorsque, expliquant que «sur un modèle mathématique de spirale autour d'un cône, [...] Pascal construit une « gradation » où les opinions se succèdent « du pour au contre ", il assignait pour terme à cette ascension, assimilée à une "conversion spirituelle ", la conquête de l'unique "point haut de la sagesse politique », celui de la reconnaissance de «l'ordre de Dieu » comme «seul bien-fondé de l'obéissance civique » - par quoi Pascal retrouverait ainsi «le sens propre de la légitimité27 ». Or il est remarquable que les commentaires les plus contemporains donnent l'impression d'aller à l'encontre d'une telle interprétation; c'est peut-être qu'ils attendent moins de Pascal la mise à jour des fondements de « la légitimité » que la description sans fard des mécanismes de la légitimation de l'ordre établi. Si Gérard Ferreyrolles souligne en termes relativement généraux que Pascal, "aussi éloigné de Savonarole qu'il l'est de Hobbes", reconnait suffisamment l'autonomie du politique pour rejeter "l'augustinisme, qui souhaite asservir l'État à l'Église ${ }^{28}$ ", Pierre Force reprend pour son compte l'interprétation des fragments 89-95, pour souligner que « dans le royaume de la concupiscence, le point de vue des habiles n'est dépassable par aucun autre », de sorte que, pour peu qu'on sache avec Pascal ne pas chercher à inscrire le champ d'une problématique politique dans l'horizon que fixent les principes de la charité, « un point de vue supérieur au point de vue des habiles ne peut être qu'un point de vue extérieur ${ }^{29}$ ». Mais Christian Lazzeri va 
encore plus loin, et paraît prêt à expulser le chrétien parfait du cône, ou du trône, sur lequel Pierre Magnard semblait vouloir le jucher : de l'interprétation de la théorie des ordres développée dans Force et justice, il résulte en effet, non seulement que la politique de Pascal peut être " considérée indépendamment du point de vue du chrétien parfait ${ }^{30}$ " mais que, même si l'on en tient compte, "la position supérieure qu'occupe le chrétien parfait dans la série des renversements du pour au contre voit sa souveraineté contrariée ${ }^{31}$ ». Cette lecture peut sembler, dans un premier temps, contredire le sens obvie du fragment 90; mais avant de s'en alarmer il conviendrait d'examiner plus à loisir l'hypothèse herméneutique originale qui la sous-tend: celle de l'entrecroisement, dans le texte des Pensées, de deux théories de la justice - non absolument conciliables.

\section{3/ Cohérence et effets de la politique pascalienne}

Christian Lazzeri se singularise en effet par sa faible inclination pour une histoire des doctrines conçue comme l'imposition zélée d'une cohérence systématique parfaite (celle par exemple d'un "ordre des raisons ») à une pensée vivante. C'est ce qui le conduit à mettre en lumière l'existence, chez Pascal, de deux théories de la justice, distinguables à défaut d'être expressément distinguées. Exposons pour commencer celle qui correspond au point de vue du chrétien parfait : pour celui-ci, même si les lois fondamentales de l'État ne doivent rien à la dévotion des princes, et tout à la décision de la force, il n'empêche que toute référence à une essentielle justice n'est pas forclose ; considérant avec saint Paul que toute puissance vient de Dieu, il reconnaît l'existence, au-delà du tableau de justice que présente l'ordre politique institué, d'une justice " extérieure et supérieure », qui, et c'est là l'important, « oblige à obéir et à honorer le pouvoir en conscience ${ }^{32}$ ». Or on peut opposer à cette théorie providentialiste de la justice une autre doctrine, fondée sur la théorie des ordres, et qui n'irait donc pas plus loin que la reconnaissance de la justice intrinsèque à l'établissement, et du droit des grands à des respects d'établissement - ce qui ne signifie pas une obéissance passive, ni absolue. On voit déjà, avec l'allusion au problème du fondement et des limites éventuelles de l'obéissance, que l'interprétation ne peut faire l'économie d'une réflexion sur les effets politiques de la pensée pascalienne.

Il y a en effet, comme l'écrit Vincent Carraud, " un effet de la raison des effets ${ }^{33}$ ", et peutêtre même plusieurs, sur le plan politique. Le premier, d'apparence paradoxale, serait selon Christian Lazzeri de contraindre ceux qui savent (c'est-à-dire, chacun dans son ordre, l'habile et le chrétien parfait) à une "politique $d u$ secret $^{34} »$ : si les croyances illusoires du peuple étaient dissipées, et les raisons (valables) de l'obéissance rendues publiques, la guerre civile surviendrait. "Étrange politique », souligne Christian Lazzeri, même si elle est la seule qui puisse maintenir la concorde intérieure, que celle qui tient secrètes les raisons d'approuver l'ordre établi, et dont en somme " tout le secret est qu'il n'y a rien à savoir ${ }^{35}$ ». La question serait alors de savoir jusqu'à quel point le chrétien parfait (ou visant à le devenir) pourrait envisager de lever un coin du voile : « Ne faut-il pas que ce chrétien s'abstienne d'écrire une Apologie de la religion chrétienne qui rendrait publique la nécessité de garder le secret relatif à l'arbitraire du pouvoir ${ }^{36}$ ? ». D’être écrite ne rend-il pas la liasse $V$ passible du soupçon de demi-habileté ? Quoi qu'il en soit de cet " effet », si important pour la détermination du statut « littéraire » et des «stratégies » rhétoriques ou argumentatives des fragments sur la "raison des effets", et de leur possible mise en abyme, on peut avoir profit, d'un point de vue plus directement politique, à examiner de plus près la question de l'obéissance et l'idée d'une théorie de la 
justice calquée sur la théorie des ordres. Dès lors qu'on préserve l'autonomie du champ politique en mettant entre parenthèses les principes de la charité, il apparaît en effet que nulle tyrannie (définie comme l'outre passement délibéré ou inconscient des limites séparant les ordres [fr. 58]) ne peut, non seulement se présenter comme légitime, mais même espérer quelque succès. La Bible refermée, nulle raison de légitimer par le recours à l'idée d'une justice transcendante ou d'une pénitence méritée les caprices d'un prince qui voudrait être estimé, ou imposer ses goûts artistiques: "La théorie des ordres de justice, résume Christian Lazzeri, résiste à celle du chrétien parfait » et "rend [...] aux gouvernés ce que celle du chrétien parfait leur enlève ${ }^{37} »$. On peut de cette manière expliquer la nécessité pour ainsi dire physique de la résistance à la tyrannie, ce qui dispense même d'en discuter la légitimité ; et s'attacher à dresser la liste des « libertés » qui devront être considérées somme de fait " incompressibles ${ }^{38}$ ", sorte de relevé des limites du pouvoir des rois qui ne serait pas moins contraignant qu'une déclaration des droits des sujets : « Les sujets ne peuvent acquiescer à toute tentative d'imposer par coercition un pouvoir sans justification, une religion, une doctrine, une esthétique officielles; ils ne peuvent accepter l'esclavage économique. [...] Ils peuvent résister à toute interdiction visant à empêcher les effets de la logique interne d'un ordre quelconque ${ }^{39}$ ». Si l'analyse de la coutume explique les procédures de légitimation de l'ordre établi, la théorie des ordres (de justice) permet de déterminer a priori les limites de la domination.

Pascal serait donc un penseur, sinon du droit de résistance, du moins des causes de l'insubordination. Au-delà de cette description quelque peu statique des rapports de pouvoir, ne pourrait-on pas cependant le créditer d'une réflexion originale sur leur évolution, voire d'une théorie normative du changement social ? Pierre Magnard croyait pouvoir parler d'un "pessimisme politique» de Pascal ${ }^{40}$, dans le droit fil d'un Lucien Goldmann pour qui « [selon Pascal] aucun changement de l'ordre social ne saurait amener la plus légère amélioration ${ }^{41}$ ». Or Gérard Ferreyrolles, sur ce point aussi, propose un renversement de perspective. Non seulement, en effet, la vision pascalienne de l'ordre publique n'est pas tragique, puisque la piperie et l'imagination assurent au royaume de concupiscence une paix bénéfique - de sorte qu'il faut y voir, plutôt qu'une «fausse image » (fr. 210) ou une "figure parodique » (Laurent Thirouin) ${ }^{42}$ de l'ordre de Dieu, un véritable "tableau de charité », admirablement réglé (fr. 118) - mais on pourrait même admettre que Pascal ne croit pas tout progrès impossible, voire affirmer qu'« en vérité, il y a un réformisme pascalien ${ }^{43} »$. De fait, remarque Gérard Ferreyrolles, comment expliquer que Pascal ait pu donner à ses amis l'impression qu'« il n'y avait rien à quoi il désirait plus de contribuer » - jusqu'à envisager, le cas échéant, d'y sacrifier sa vie - que l'instruction d'un prince (fr. 1004), s'il avait « [estimé] impossible la moindre amélioration de l'ordre politique social ${ }^{44} »$ ? Or à ce propos attribué à Pascal, qu'allègue Gérard Ferreyrolles, on pourrait ajouter, comme un autre témoignage du réformisme pascalien, la conclusion des Discours sur la condition des grands. « Ne prétendez donc point [...] les traiter avec dureté. Contentez leurs justes désirs ; soulagez leurs nécessités » : on est loin ici du despotisme, et même du conservatisme. Outre qu'il formule les règles d'une méthode de gouvernement, Pascal trace ici la ligne directrice du changement social: " avancez-les autant que vous pourrez ${ }^{45}$ ». Certes, on peut penser que ce réformisme éclairé n'est pas démocratique ${ }^{46}$, puisque c'est à un roi, et non à ses sujets, que ce discours s'adresse. Le peuple ne pourrait-il être rendu habile? De fait, «le peuple n'est pas susceptible de cette doctrine ", lisons-nous au fr. 525. À quoi le fr. 66 (qui dans l'économie du discours XV re[con]stitué par Emmanuel Martineau vient du reste à la suite du fr 525 ${ }^{47}$ ) apporte cependant une correction essentielle : il ne s'agit plus en effet de ne rien dire au 
peuple, mais de «lui dire en même temps" une vérité de fait («les lois ne sont pas justes») et la vérité politiquement fondamentale («il y faut obéir parce qu'elles sont lois »). Entorse de taille à la " politique du secret ", qui ne cède même pas le pas, ici, à la définition d'une pratique du double langage, mais bel et bien à une pédagogie politique, assez bien accordée avec les objectifs réformistes par ailleurs assignés par Pascal à la politique d'un vrai roi de concupiscence. Et certes, le chrétien parfait pourrait accueillir avec une condescendance mêlée d'effroi cette ambition normative et programmatique, obéissant à des impératifs socio-politiques plutôt qu'aux principes de la charité : «Si vous en demeurez-là, vous ne laisserez pas de vous perdre ${ }^{48}$. À quoi l'habile pourrait toujours répondre, comme en se jouant, qu'après tout « il y a des gens qui se damnent si sottement ${ }^{49}[\ldots] ! »$.

\section{II- Force de la justice et violence symbolique : tentatives contemporaines d'appropriation de Pascal}

\section{1/ La déconstruction à la limite}

Les historiens de la philosophie feront un jour un relevé des similitudes que la méthode ou le style général de la "déconstruction» derridienne présentent avec le mode de raisonnement introduit par Pascal sous le titre de "raison des effets ». Cependant, s'il a $\mathrm{pu}$ arriver à Jacques Derrida, dans un livre récent, de s'intéresser de près au texte pascalien, c'est pour des raisons beaucoup plus précises, tenant au développement de sa propre réflexion, et notamment à la place de plus en plus centrale qu'y tient la question des sources et de la déconstructibilité du droit, lui-même opposé à une irréductible « justice », objet d'une promesse infinie. Si Jacques Derrida va à la rencontre de Pascal, c'est dans sa recherche d'une justice qui « excède ou contredit le droit ", et se révèle « indéconstructible » du fait même qu'elle se situe « hors ou au-delà du droit ${ }^{50}$ ». Car cette justice-là n'est pas antagoniste de la force, elle l'exige au contraire, plus fondamentalement encore que ne le fait la « justice », telle qu'on l'entend communément, c'est-à-dire réduite à la force autorisée du droit.

Le recours à la liasse $\mathrm{V}$ des Pensées, et plus précisément au fr. $103^{51}$, est donc pour Jacques Derrida une façon de souligner la dette originaire de la justice à l'égard de la force. Le « il faut » de la formule pascalienne (« il faut donc mettre ensemble la justice et la force ») signifierait au moins que « la nécessité de la force est impliquée dans le juste de la justice ${ }^{52}$ ». Toutefois Jacques Derrida s'efforce de proposer de ce fragment fameux une interprétation qu'il présente comme non traditionnelle, et dont il précise qu'elle risque d'apparaître comme " tout sauf non-violente ${ }^{53}$ ». L'interprétation " conventionnaliste " de Pascal, transformé moyennant un rapprochement avec Montaigne en porte-parole d'un "scepticisme pessimiste, relativiste et empiriste $\mathrm{e}^{54}$ ». Or, à en croire Jacques Derrida, «la pensée de Pascal qui « met ensemble » la justice et la force et fait de la force une sorte de prédicat essentiel de la justice [...] va peut-être au-delà d'un relativisme conventionnaliste ou utilitariste, au-delà d'un nihilisme, ancien ou moderne [...], au-delà de la morale cynique du Loup et l'Agneau ${ }^{55}$ ». Cette "pensée » mettrait en évidence, en effet, une relation pouvoir-violence plus fondamentale et plus «interne " que celle à laquelle on fait allusion lorsqu'on crédite Pascal de l'aperçu « moderne » suivant lequel le droit serait au service d'une (injuste) violence de classe ou d'État. Ce que Pascal aurait en vue, c'est plus profondément la «violence performative» ou le coup de force que 
représente l'opération consistant à "faire la loi ", violence qui tient à ce que nulle loi d'avant la loi, nul «droit préalable » issu d'une «fondation préexistante ${ }^{56}$ » ne pourrait légitimer cette institution. Cette violence, ni juste ni injuste, sans laquelle il n'y aurait ni justice ni injustice, déploie une logique paradoxale assimilée par Jacques Derrida à celle des énoncés performatifs étudiés par Austin : « aucun discours justificateur ne peut ni ne doit assumer le rôle de métalangage par rapport à la performativité du langage instituant 57 ».

Les fragments 103 (et 60) des Pensées mettraient donc en évidence, au-delà de la déconstructibilité du droit, l'indéconstructibilité d'une archi-justice auto-fondée. Or tout l'effort de Jacques Derrida est à partir de là de montrer que la violence intrinsèque à la justice, loin de se manifester seulement à son origine, trouve un écho ou un prolongement singulier en toute décision quant au juste et à l'injuste. Si le droit est en effet pour Jacques Derrida, ici très proche d'un certain décisionnisme, de l'ordre de la raison calculante, et semble ne requérir que l'application réglée de normes, il serait en revanche ridicule de réduire le juge à une " machine à calculer ", et d'oublier que la décision de justice n'a pas seulement pour effet de conserver la loi en l'appliquant, mais suppose au contraire, comme la condition de son application à un cas singulier, que la loi soit suspendue ou provisoirement déniée pour être, à chaque fois, « réinvent[ée], rejustifi[ée], réinvent[ée] au moins dans la réaffirmation et la confirmation nouvelle et libre de son principe $^{58}$ ». Une décision ne peut être juste qu'à témoigner de l'incalculable, ou si l'on préfère à affronter l'indécidable. La décision, qu'aucune règle n'assure, est l'affirmation affolante d'un « appel de la justice ${ }^{59}$ » qui, en tant qu'ouverture à un avenir indéterminé, soustrait à tout horizon d'attente (« la justice reste à venir, elle $a$ à venir, elle est à venir $\left.{ }^{60} »\right)$, réitère indéfiniment ce coup de force de la loi que Pascal pouvait donner l'impression de considérer comme déjà advenu, une fois pour toutes.

\section{2/ Pascal, précurseur de la sociologie?}

17 Les fragments de la liasse V (et connexes) n'intéressent pas seulement, outre leurs exégètes «spécialisés ", les philosophes soucieux de démontrer pour se défendre contre des contresens intéressés que "les "déconstructionnistes" « ont bien "quelque chose à dire sur la justice, quelque chose à faire avec la justice ${ }^{61} »-$ ne serait-ce qu'en tant que la justice, seule, serait indéconstructible, tandis que la déconstruction n'en appellerait à rien plus obstinément qu'à la justice. Ces mêmes fragments peuvent également, aujourd'hui, être employés par la sociologie comme autant d'instruments, utilisables notamment pour dénoncer les travers d'un discours philosophique soupçonné d'être, dans sa prétention à l'universalité et au désintéressement, enclin à l'emphase normative autant qu'à la morgue aristocratique. Pascal, pour indisposer (à) la philosophie? Il faudrait presque en faire un sociologue de rencontre pour pouvoir le transformer en allié privilégié d'une certaine antiphilosophie: j'ai, confessait récemment Pierre Bourdieu, " toujours su gré à Pascal [...] de sa sollicitude, dénuée de toute naïveté populiste, pour le " commun des hommes » et les « opinions du peuple saines »; et aussi de sa volonté, qui en est indissociable, de chercher toujours la "raison des effets ", la raison d'être des conduites humaines en apparence les plus inconséquentes ou les plus dérisoires [...], au lieu de s'en indigner ou de s'en moquer, à la manière des « demi-habiles », toujours prêts à « faire les philosophes » et à tenter d'étonner par leurs étonnements hors du commun à propos de la vanité des opinions du sens commun ${ }^{62}$ ». Philosophes demi-habiles dont Pierre Bourdieu ajoute beaucoup plus loin que «faute de s'interroger sur la « raison des 
effets » qui suscitent leurs étonnements, ils contribuent à détourner des réalités les plus dignes de provoquer l'étonnement ${ }^{63} »$.

C'est donc d'abord une leçon de style et de méthode que la sociologie peut recevoir du Pascal de la liasse V. L'influence de Pascal sur Pierre Bourdieu est du reste si prégnante qu'on a parfois l'impression d'une quasi-identification du disciple au maître - par exemple au vu de cette autre confession: "S'il m'arrive de sasser et de ressasser les mêmes thèmes, de revenir à plusieurs reprises sur les mêmes objets et les mêmes analyses, c'est toujours, il me semble, dans un mouvement en spirale qui permet d'atteindre à chaque fois un degré d'explicitation et de compréhension supérieur, et de découvrir des relations inaperçues et des propriétés cachées ${ }^{64} \ggg$. En allant au-delà des considérations méthodologiques, on peut cependant s'intéresser à la manière dont les textes pascaliens ayant trait à la «raison des effets» sont mis au service de la polémique antiphilosophique. C'est, pour commencer, le fragment $60^{65}$ qui se trouve mobilisé. Pierre Bourdieu en retient surtout le rôle prééminent de la coutume, assimilée à un " principe de déraison suffisante", lequel suffit en tout cas à légitimer une pétition de principe historiciste: ce que voudrait dire Pascal, c'est à l'origine du droit il y a, plus fondamentalement encore que la violence, «l'arbitraire historique de l'institution historique $^{66}$ ». Cette vérité première autorise selon Pierre Bourdieu à rejeter dans leur totalité, en tant que purs et simples «mythes d'origine des religions démocratiques », les «théories du contrat ». Vouée aux gémonies sans la moindre tentative de discussion ni même la précaution d'aucune citation, la "Théorie de la justice de John Rawls", qui n'aurait à son tour rien fait d'autre qu'essayer de conférer « leur lustre de rationalité » à ces « religions », est elle aussi promptement évacuée au nom d'une pascalienne lucidité : «Rien n'est [...] plus vain, en ces matières, que l'ambition de la raison qui prétend se fonder elle-même, en procédant par déduction rigoureuse à partir de " principes $^{67}$ » ». Mais du fragment 60 , comme d'autres qui vont dans le même sens, Pierre Bourdieu retient aussi l'indication des limites de la rationalité discursive au regard du pouvoir décisif de la force. C'est, du coup, Jürgen Habermas qui se voit reprocher de n'avoir pas voulu voir que «la force des arguments n'est guère efficace contre les arguments de la force", et que «la domination n'est jamais absente des rapports sociaux de communication ${ }^{68} »$. Au reste, et plus généralement, c'est l'oubli plus ou moins délibéré de la réalité du monde social qui semble à Pierre Bourdieu une constante ou un présupposé tacite du discours philosophique. Or la vérité serait au contraire que " "nous sommes embarqués", comme dit Pascal ${ }^{69} »$ - c'est-à-dire enfermés dans le cadre prédéfini des habitus d'un "champ» où nous n'avons pas décidé de nous inscrire. De sorte que là encore Pascal peut servir d'antidote à l'illusion philosophique de la liberté dans le « choix du métier ", si l'on veut bien admettre du moins que sa théorie des ordres est transposable telle quelle à la description sociologique des «champs »: "Chaque champ, comme l'ordre pascalien, enferme aussi les agents dans ses enjeux propres qui, à partir d'un autre point de vue, c'est-à-dire du point de vue d'un autre jeu, deviennent invisibles ou du moins insignifiants ou même illusoires ${ }^{70}$ ». Ainsi des enjeux de la philosophie tels qu'envisagés sous l'angle sociologique.

19 Toutefois, si Pascal peut faire figure de précurseur de la sociologie autant que d'allié dans la polémique antiphilosophique, c'est principalement au titre de sa contribution à l'étude du fonctionnement objectif des champs sociaux, et notamment des modalités et des moyens de la domination. S'il est vrai que "la coutume fait toute l'autorité » (sic: c'est ainsi que Pierre Bourdieu cite, entre guillemets, le fr. 60), on peut en déduire qu'aux yeux de Pascal 
«l'accoutumance à la coutume et à la loi que la loi et la coutume produisent par leur existence et leur persistance mêmes suffit pour l'essentiel [...] à imposer une reconnaissance de la loi fondée sur la méconnaissance de l'arbitraire qui est en son principe $^{71}$ ». Là encore, le sociologue Pascal a raison contre les philosophes : nul besoin, par exemple, d'invoquer l'action d'« appareils idéologiques d'État » pour rendre compte de la soumission des sujets à la loi ${ }^{72}$. Plus largement, c'est tout «ce que l'on met d'ordinaire sous le concept d'idéologie » qui voit son importance remise en cause : c'est à même les corps, et non dans les esprits, que s'inscrivent les effets de la violence symbolique. La soumission repose sur « une croyance tacite et pratique rendue possible par l'accoutumance qui naît du dressage des $\operatorname{corps}^{73}$ ». La «tradition marxiste» se tromperait donc fondamentalement, cédant après tant d'autres ${ }^{74}$ à "l'illusion scolastique ", dans sa tentative de "décrire la résistance à la domination dans le langage de la conscience ${ }^{75}$ ». Le matérialisme historique est mis dans le même sac que toute la «tradition intellectualiste des philosophies de la conscience », coupable de n'avoir pas su expliquer « la soumission doxique des dominés » à l'ordre social ${ }^{76}$.

Il est pourtant remarquable qu'en dépit des ambitions purement descriptives et explicatives du "savant ", le souci de Pierre Bourdieu soit aussi, comme, on l'a vu, celui de Jacques Derrida et de la plupart des commentateurs actuels de Pascal, de relire les analyses pascaliennes visant à déceler la "raison des effets " à la lumière d'une revendication quelque peu indéterminée, mais néanmoins inconditionnelle, de justice ou d'émancipation. Néanmoins, Pierre Bourdieu ne cherche pas sur ce point à prendre directement appui sur des textes de Pascal : celui-ci, à l'en croire, se serait limité au « pur constat [...] de l'arbitraire originel ${ }^{77} »$. Pour le coup, ce serait donc à la seule sociologie, affranchie des limites dans lesquelles s'enfermait le propos pascalien, de tâcher de propulser dans le champ politique des « porte-parole des dominés » disposant, au contraire de ces derniers, "des instruments d'expression et de critique» sans lesquels nul ne saurait «s'arracher à l'évidence silencieuse de la doxa ${ }^{78}$ ". Ces "professionnels de l'explication » mettraient alors leur précieux « capital culturel » au service des dominés, incapables sinon « d'accéder à la mobilisation collective et à l'action subversive contre l'ordre symbolique établi ${ }^{79}$ ». Il ne nous incombe pas, bien sûr, de juger de la validité et de la pertinence des perspectives théoriques et politiques qu'ouvre à Pierre Bourdieu sa tentative d'appropriation et de dépassement de la problématique pascalienne de la « raison des effets ». À coup sûr, pourrions-nous être tentés de noter charitablement, elle échappe au piège de la "naïveté populiste » redoutée aujourd'hui par Pierre Bourdieu. Mais peut-être pas au vertige d'une certaine illusion («scolastique»?) de supériorité, dont peut toujours être saisi l'intellectuel «compagnon de route» dès lors qu'il s'improvise chef de cohorte. Comme si une même question devait en somme nécessairement être adressée à la reprise sociologique de la pensée de Pascal, mais aussi bien aux autres commentaires et interprétations qui s'attardent à en examiner les attendus et les conséquences - voire comme le suggère Christian Lazzeri à l'auteur de la liasse V lui-même : qui peut être sûr d'avoir atteint à la parfaite habileté ? Comment éviter de n'apparaître pas, dès lors qu'on s'exprime sur ces matières, comme le demihabile d'un autre? Pour ne rien perdre de son habileté, il faut peut-être garder le silence. Mais comment ne pas parler? 


\section{NOTES}

1. Pierre Bourdieu, Méditations pascaliennes, Paris, Seuil, 1997, p. 214.

2. «La politique de Pascal existe, [...] elle est cohérente et [...] original »: Pascal et la raison du politique, Paris, P.U.F., 1984, p. 5.

3. V. par exemple Laurent Thirouin, «Le réalisme de Pascal », in Justice et force, Actes du colloque de Clermont-Ferrand, Paris, Klincksieck, 1996.

4. Le même Laurent Thirouin parlait naguère de «cynisme », en ajoutant : «La pensée de Pascal se fait parfaitement immorale à force de réalisme » («Raison des effets, essai d'explication d'un concept pascalien ", Dix-septième siècle, $\mathrm{n}^{\circ} 134$, janvier-mars 1982, p. 47).

5. "...aux conservateurs pour ce qu'il met l'usurpation à l'origine de l'autorité et aux révolutionnaires pour ce qu'il prêche la soumission » (op. cit., p. 10).

6. Idem.

7. Pierre Magnard, Nature et histoire dans l'apologétique de Pascal, Paris, Les Belles Lettres, 1975, p. 235.

8. Gérard Ferreyrolles, op. cit., p. 188.

9. Id., pp. 189 et, pour la dernière phrase citée, 195. Gérard Ferreyrolles met ainsi Pascal d'accord avec Arnauld et Domat.

10. Id., p. 190.

11. Gérard Ferreyrolles, Les Reines du monde, L'imagination et la coutume chez Pascal, Paris, Champion, 1995, p. 88.

12. Pascal et la raison du politique, p. 197.

13. Cf. a contrario Spinoza, Éthique, Quatrième partie, scolies des propositions XVIII et XIX.

14. Christian Lazzeri, Force et justice, Paris, P.U.F, 1993, p. 203, en note (la discussion se poursuit jusqu'au bas de la p. 205).

15. Id., p. 203. Cf. p. 202 sur le contraste entre les positions de Pascal et Arnauld.

16. Christian Lazzeri, Force et justice, p. 244.

17. Tandis que les normativismes d'inspiration religieuse avaient surtout fait la preuve de leur capacité à plonger le pays dans «le chaos d'une guerre civile sans vainqueurs ni vaincus, à laquelle d'ailleurs la seule décision politique de l'État mit fin » (Carl Schmitt, «La formation de l'esprit français par les légistes", traduction française Jean-Louis Pesteil, in Du politique, Puiseaux, Pardès, 1990, p. 200 sq.).

18. Car Schmitt, Théologie politique (1922), traduction française Jean-Louis Schlegel, Paris, Gallimard, 1988, p. 15. La « décision de l'exception » est la décision éminente, car elle ne se laisse déduite d'aucune norme juridique en vigueur (elle les suspend toutes, au contraire) ; c'est la décision absolue.

19. Fragments 281 et 280 de l'édition Lafuma (c'est moi qui souligne).

20. Christian Lazzeri, Force et justice, p. 246.

21. Idem (je souligne).

22. Christian Lazzeri, Force et justice, p. 247.

23. Justice et force, collectif cité, p. 344. De là à conclure avec Jean Mesnard que la force est "neutre ", comme si elle ne forçait pas (à) la décision, il y a un pas sans doute infranchissable. On pourrait dire de la force, comme Carl Schmitt le faisait de la technique, que loin d'être neutre elle emporte avec elle l'exigence d'une politique assez forte pour se l'assujettir (v. "L'ère des neutralisations et des dépolitisations " (1929), in La notion de politique, traduction française de Marie-Louise Steinhauser, Paris, Calmann-Lévy, 1972, p. 152 sq.). 
24. Pierre Force, «Pascal et Machiavel », in Justice et force (collectif cité), p. 63.

25. Idem.

26. Carl Schmitt, Théologie politique (op. cit), p. 23.

27. Pierre Magnard, Nature et histoire (op. cit.), p. 241 à 243.

28. Gérard Ferreyrolles, Pascal et la raison du politique (op. cit.), p. 228.

29. Pierre Force, Le problème herméneutique chez Pascal, Paris, Vrin, 1989, p. 237 sq.

30. Christian Lazzeri, Force et justice, (op. cit.), p. 258.

31. Christian Lazzeri, Force et justice, (op. cit.), p. 353.

32. Idem, p. 349.

33. Vincent Carraud, Pascal et la philosophie, Paris, P.U.F., 1992, p. 260.

34. Christian Lazzeri, Force et justice, (op. cit.) p. 349.

35. Idem, p. 333.

36. Idem, p. 349.

37. Idem, p. 352.

38. Idem, p. 353.

39. Idem.

40. Pierre Magnard, Nature et histoire... (op.cit.) p. 229.

41. Lucien Goldmann, Le Dieu caché, Paris, Gallimard, rééd. « Tel », 1976, p. 307.

42. Laurent Thirouin, «Raison des effets, essai d'explication d'un concept pascalien» (art. cit.), p. 48. C'est, à ce compte, une « raison effrayante» (je souligne) que celle qui « structure la société humaine » (Idem).

43. Gérard Ferreyrolles, Pascal et la raison du politique (op. cit.) p. 194.

44. Idem.

45. Pascal, troisième Discours sur la condition des grands, éd. Lafuma, Paris, Seuil, «L'intégrale ", 1963, p. 368.

46. Sur les similitudes, en revanche, entre la conception rousseauiste de la démocratie et l' ecclésiologie (non la politique) pascalienne, v. les remarques très suggestives d'E. Martineau (à opposer à l'interprétation organiciste de Gérard Ferreyrolles, Pascal et la raison du politique, p. 246), in « Deux clés de la chronologie des discours pascaliens », XVII ${ }^{e}$ Siècle, 1994-4, p. 728.

47. Gérard Ferreyrolles donne quant à lui le dernier mot au fr. 525, contre le fr. 66, pour conclure à la vanité de toute « démystification " possible en politique (Pascal et la raison du politique, p. 119).

48. Pascal, troisième Discours sur la condition des grands, éd. Lafuma, Paris, Seuil, «L'intégrale », 1963, p. 368.

49. Idem.

50. Jacques Derrida, Force de loi, Paris, Galilée, 1994, respectivement p. 17 et 35.

51. Jacques Derrida cite Pascal dans l'édition Brunschvicg (fr. 298).

52. Jacques Derrida, Force de loi, p. 31.

53. Idem

54. Idem.

55. Jacques Derrida, Force de loi, p. 31.

56. Idem, p. $32 \mathrm{sq.}$

57. Idem, p. 33.

58. Idem, p. 51. Cf. Carl Schmitt, Théologie politique (op. cit.), p. 41 sq.

59. Jacques Derrida, Force de loi, p. 38

60. Idem, p. 60.

61. Idem, p. 14.

62. Pierre Bourdieu, Méditations pascaliennes, Paris, Seuil, 1997, p. 10.

63. Idem, p. 214.

64. Idem, p. 17 (je souligne).

65. Selon la numérotation Lafuma (Pierre Bourdieu cite d'après l'édition Brunschvicg). 
66. Pierre Bourdieu, Méditations pascaliennes, p. 114.

67. Idem, p. 114 sq.

68. Idem, p. 80.

69. Idem, p. 23.

70. Idem, p. 117.

71. Idem, p. 201.

72. Idem.

73. Idem, p. 205. Pour une explicitation de cette critique voilée de Marx, cf. le scolie 2 de la proposition 0 des «Fondements d'une théorie de la violence symbolique », in Pierre Bourdieu / Jean-Claude Passeron, La reproduction, Paris, Minuit, 1970, p. 18 sq.

74. Par exemple, « il faut rompre avec l'intellectualisme de la tradition kantienne » (p. 210).

75. Méditations pascaliennes, p. 205.

76. Idem, p. 211.

77. Idem, p. 128 (je souligne).

78. Idem, p. 224.

79. Idem.

INDEX

Mots-clés : Pascal, Pensées, raison des effets, politique, justice, philosophie, sociologie, Derrida (Jacques), Bourdieu (Pierre)

Keywords : reason, effect, politics, philosophy, sociology

\section{AUTEUR}

\section{PHILIPPE DUCAT}

Faculté des Lettres de l'Université de Pau et des Pays de l'Adour 